\title{
Role of Physical Activity and Nutrition on Brain Health in Older Adults Based on Assessment
}

\author{
Chiyuri Nagayama ${ }^{1,2}$ \\ 1. Graduate School of Ochanomizu University, Tokyo 112-0012, Japan \\ 2. Tokyo Metropolitan Senior High School, Tokyo 156-0055, Japan
}

Received: September 22, 2013 / Accepted: October 22, 2013 / Published: December 31, 2013.

\begin{abstract}
By day-to-day rehabilitation, health status of the people will continue to improve. Therefore, rehabilitation is a method of sustainable social participation. It has discussed how smart community would be. It was aimed to measure the variation due to body rehabilitation in everyday life. By this, the possibility of smart community will be suggested. By measuring EMG, ankle torque and brain activity, the effect of rehabilitation was evaluated quantitatively. And also, adaptation modeling was used for the analysis. Analysis of EMG, it was possible to observe stretch reflex through the central nervous system. Compared between acute stroke and chronic stroke, the parameter of acute stroke is higher than that of chronic stroke. To taking changes in physical function throughout rehabilitation will lead to contribution to environmental issues. On the other hand, brain plasticity based on rehabilitation is further expected to education. The development process in education, physical parameters change. It was found that the measurement results of the rehabilitation, and has been deeply involved in nerve. The rehabilitation and educational issues in the everyday, people should support each other and grow with.
\end{abstract}

Key words: Sustainable community, neurorehabilitation, ubiquitous computing, healthcare.

\section{Introduction}

\subsection{Neuroplasticity of Cerebral Motor Functions}

Cortical motor representations are not static but fluid and regulated by use. The most common approach taken to investigate the potential for cortical plasticity has been to evaluate the reorganization of sensory and motor maps following peripheral or central lesions and compare them to normal animals. In these studies either the receptive field properties or the kinds of movements evoked by electrical stimulation are examined over a large set of sites in the cortex. The data collected from these sites can be used to construct maps of the cortical representation pattern. The expansion or reduction in the size of an area of representation and the time course of these events are

Corresponding author: Chiyuri Nagayama, M.Sc., research field: human brain and rehabilitation. E-mail: Chiyuri.Nagayama@gmail.com. then compared. An important principle of reorganization is "Use It or Lose It". If a limb is amputated and hence, not used, the cortical representation shrinks. If limb use is increased, for example, in learning a new motor skill, the representation increases. Research has increasingly become focused on the synaptic mechanisms underlying this plasticity in order to apply the findings to rehabilitation of stroke and neurological disorders. Recent experimental research is elucidating the synaptic mechanisms underlying cortical reorganization and the effect of therapy on this process.

Motor cortical plasticity, shown in Fig. 1, has recently been documented with experience, enabling the study of "Learning and Memory" and how the brain changes with experience. Deficit of motor function is recovered by sustainable passive motion. At the simplest end of the behavioral spectrum, changes in “Environment” can alter cortical representations and 


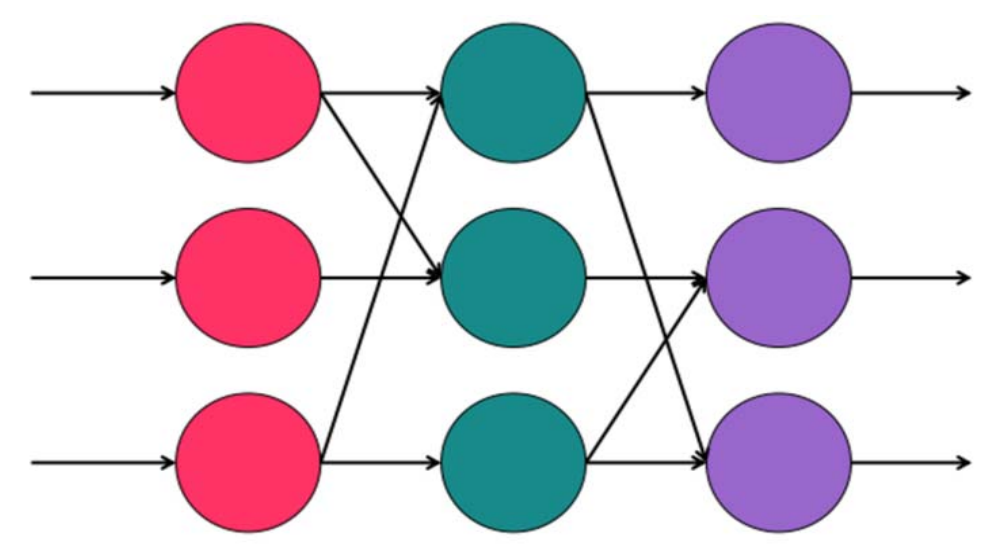

Fig. 1 Neuronal network for effect of neurorehabilitation to lower limb.

improve behavioral recovery after injury. Rats exposed to an enriched environment after focal ischemic strokes improved more in sensorimotor tasks than did rats housed in a standard environment post injury. This behavioral improvement has been attributed to plasticity of surrounding, healthy tissue.

Cortical plasticity in the motor cortex could rely on one or more of the following mechanisms: changes in balance of excitation and inhibition resulting in the unmasking of latent connections, strengthening or weakening of existing connections by LTP or LTD, generalized modulatory effects with pharmacological or state changes, or Neurogenesis (Synaptogenesis). LTP, which results in long-term changes in synaptic efficacy, in Hebbian in nature, resulting from exact temporal patterning of inputs to a target neuron. Conditional LTP especially has received much attention as a mechanism capable of reorganizing the motor cortices. Pharmacological manipulations or activation of particular afferent pathway stimulation have been shown to modify motor cortical synapses. Activity-dependent synaptic plasticity is restricted to specific synapses activated by inputs and is, therefore, very restrictive and focal, an appealing mechanisms for learning. It could explain how converging afferent inputs from disparate sources could interact in the cortex to induce plasticity.

\subsection{Neuroplasticity of Genetic Aspects}

Various forms of neuronal loss, necrosis and/or apoptosis are also neural plasticity. Therefore, the primary aim of any therapeutic interneuron is to prevent or moderate ischemic cell damage. Programmed cell death is a time consuming mechanism compared to the rapid process of postischemic necrosis. Therefore, the regular intervention including anti-apoptotic gene transfer could be a real option. Apoptosis, programmed cell death could be initiated by the activation of intrinsic (mitochondria-dependent) and extrinsic (receptor-dependent) pathways. Bcl-2 and Bcl-XL anti-apoptotic proteins inhibit intrinsic mitochondrial dependent cell death by conservation the integrity of the outer mitochondrial membrane [1]. This function prevents the release of cytochrome c from the mitochondria. The anti-apoptotic members of the Bcl-2 protein family are neuroprotective in the ischemia-induced cell death, therefore it can be assumed that Bcl-2 gene delivery into the neuron at risk prevents neuronal apoptosis. $\mathrm{Bcl}-2$ and $\mathrm{Bcl}-\mathrm{XL}$ anti-apoptotic gene transfer can decrease hypoxic impairments by reducing apoptosis. At the same time these genes increase GAP-43, one of the proteins responsible for neuronal plasticity. These interrelated actions of activated anti-apoptotic genes could be a beneficial after ischemic stroke.

Dopamine, like serotonin, is a major monoaminergic transmitter in the central nervous system. The majority of dopaminergic neurons have their cell bodies in the substantial nigra while their axons project to the corpus 
striatum. Dopaminergic neurons have been implicated in the regulation of motor behavior-the degeneration of dopaminergic neurons underlies Parkinson disease, a debilitating disorder of movement. Other dopaminergic pathways are thought to regulate motivated behaviors. Dysfunction of these pathways may contribute to Schizophrenia. The role of the dopaminergic system in mammalian behavior has traditionally been studies through pharmacological techniques. Recently, however, gene knockout techniques have been applied to this system. In one set of experiments the ability of neurons to synthesize dopamine was blocked by selectively inactivating the gene that encodes tyrosine hydroxylase, one of the enzymes important in dopamine synthesis. The dopamine-deficient mice were born, began to nurse, and grew normally for about two weeks and then became inactive, failed to eat or drink, and died shortly thereafter. However, daily administration of L-DOPA, the product of tyrosine hydroxylase, restored normal feeding and produced increased activity. Dopamine is cleared from synapse by a high-affinity dopamine transporter. In mutant mice with a deficiency in this transporter the amount of extracellular dopamine is 100 -fold greater than normal. The mutant mice exhibit spontaneous and excessive locomotion similar to that obtained in normal mice when the dopamine transporter is blocked pharmacologically.

One of the first complex human behavioral abnormalities to be traced to a single gene is Huntington disease, a degenerative disorder of the nervous system [2]. Huntington disease affects both men and women with a frequency of about 5 per 100,000 . It is characterized by four features: heritability, chorea, cognitive impairment and death 15 to 20 years after the onset of symptoms [3]. In most patients the onset of the disease occurs in the fourth to fifth decade of life. Thus, the disease often strikes after individuals have married and had children. The mutation causing HD is an expanded tract of glutamine residues in the first exon of the Huntington gene product $(H t t)$, a 350-kDa protein of unknown function.

\subsection{Neuroplasticity of Auditory Functions}

Human experience is enriched by our ability to distinguish a remarkable range of sounds-from the complexity of a symphony, to the warmth of a conversation, to the dull roar of the stadium. This ability depends upon the almost miraculous feats of hair cells, the receptors of the internal ear. Similar hair cells are also responsible for our sense of equilibrium [4]. Human hearing commences when the cochlea, the snail-shaped receptor organ of the inner ear, transduces sound energy into electrical signals and forwards them to the brain. The cochlea, however, is not simply a passive detector. The ability to recognize small differences in sounds stems from the capacity of auditory system to distinguish among frequency components and to inform of both the tones present and their amplitudes. The cochlea also contains cellular amplifiers that augment auditory sensitivity and are responsible for the first stages of frequency analysis.

The paired cochlea contains slightly more than 30,000 receptor cells. These hair cells carry out the process of auditory transduction: They receive mechanical inputs that correspond to sounds and transduce these signals into electrical responses that can be forwarded to the brain for interpretation. Hair cells can measure motions of atomic dimensions and transduces stimuli ranging from static inputs to those at frequencies of tens of kilohertz. Damage to or deterioration of hair cells accounts for most of the hearing loss in the nearly 30 million Americans who are afflicted with significant deafness. Information flows from the cochlea to the cochlear nuclei, which signals ascend the brain stem through a richly interconnected series of relay nuclei. The brain stem components are essential for localizing sound sources and suppressing the effects of echoes. The auditory regions of the cerebral cortex further analyze auditory information and deconstruct complex sound patterns such as human speech. 
Auditory cortex plays a crucial role in higher perceptual and cognitive functions, including those of speech and music, and in the processing of auditory space. Cortical plasticity, as in other sensory systems, is used to fine-tune these higher functions and plays an important role in reorganization after early injury. Auditory cortical plasticity can be demonstrated after lesions of the cochlea and appears to participate in generating tinnitus. Early musical training leads to an expansion of auditory cortex representing complex harmonic sounds. Similarly, the early phonetic environment has a strong influence on speech development and, presumably, cortical organization of speech. In auditory spatial perception, the spectral cues generated by the head and outer ears vary individually and have to be calibrated by learning. The neural mechanisms of plasticity are likely the same across cortical regions. It will be useful, therefore, to relate some of the findings and hypotheses in auditory cortical plasticity to previous studies of other sensory systems.

Research on the auditory system has been much slower to recognize the relevance of cortical self- and re-organization than research in the other major sensory systems that are vision and touch. Although there have been a number of studies of auditory plasticity up to the level of the inferior colliculus, most work on plasticity of auditory cortex is comparatively recent. Many important functions of the auditory system, however, can only be fully understood with cortical plasticity in mind. For instance, even with an innate capacity for language, normal speech can hardly be acquired without auditory feedback and a capacity for learning [5]. Likewise, a system capable of localizing sound at extraordinary precision, using various sets of cues, can not accomplish this without resorting to tuning mechanisms that re-calibrate the system continually, especially during the growth phase of the head and outer ears. Spatial processing of sound and acoustic communication epitomize the two major functions of hearing. The review will concentric on auditory cortical plasticity from these two vantage points and, in surveying some of the existing literature, will make suggestions for further study.

Finally, plasticity of the auditory system has an important function in the compensation of early loss of vision and hearing [6]. When animals or humans are born or grow up blind, auditory functions expand into previously visual regions of the brain. This can be demonstrated with neurobiological and functional imaging techniques and confirms behavioral observations. Conversely, in deaf individuals auditory cortex also reorganizes to take on other functions that permit those individuals to use it for communication through other sensory modalities.

Tinnitus, the hearing of a disturbing tone or noise in the absence of a real sound source, is in many ways comparable to the experience of phantom pain, which can still be felt in an amputated limb. Tinnitus can be thought of as an auditory phantom phenomenon, in which the firing of central auditory neurons, quite possibly in the auditory cortex, still convey specific perceptual experiences, even though the corresponding sensory receptor cells that used to encode them have long been destroyed.

One of the most promising theories on the origin of tinnitus is that of a plastic reorganization in the auditory cortex following peripheral trauma caused, for instance, by loud noise exposure or aging. According to this hypothesis, the process leading to tinnitus begins with a sensorineural hearing loss in the auditory periphery. This could be a cochlear lesion from loud noise exposure or age-related hair cells loss within a certain frequency range. While the loss of hair cells causes elevated thresholds in that frequency range. While the loss of hair cells causes elevated thresholds in that frequency range, neighboring frequency portions may actually be amplified because their central representation expands into the vacated frequency range. Indeed, preliminary exists from MEG and PET for an expansion of the frequency representation in auditory cortex around the tinnitus 
frequency, indicated in Fig. 2. These results await confirmation with high-resolution fMRI (functional magnetic resonance imaging) [7]. Recent results from high-resolution structural MRI studies using voxel-based morphometry indicate the medial geniculate nucleus as another central auditory site, alternative or in addition to auditory cortex, in which this reorganization may occur. As has been shown in animal studies, the resulting loss of hair cells in a specific part of the cochlea leads to a characteristic cortical reorganization of auditory cortex. Frequency regions neighboring to the lesion part expand into the vacated space and become overrepresented. In addition, these regions lose intracortical inhibitory input from the deafferented cortical region. Cortical neurons with input from frequency ranges next to the cut-off frequency thus display permanently elevated spontaneous activity levels. Similar effects are observed after loud noise exposure or aging. It is assumed that the same synaptic mechanisms are responsible for reorganization after lesions and in tinnitus that lead to reorganization from experiential effects of auditory training. Mind resilience is such as visual and auditory neural plasticity.

Depending on the timing of the stimulation of visual stimuli and auditory stimuli, plastic change comes change. If simultaneously, the subliminal effect occasions are known. This fact confirms that the interaction of language learning and problem-based learning, such as practical is desirable in education.

\section{Materials and Methods}

The author aimed to analyze use of viscoelasticity measurement for evaluating functional recovery from stroke. The range of motion in the ankle joints of acute stroke and that of chronic stroke were compared. The range of motion is different from the level of functional recovery. Resistive torque can be separated into a passive component and an active component through analysis with numerical computation software. In the present study, the passive component and active component from this analysis in acute stroke and chronic stroke were compared. The contribution to resistive torque from the nervous system was factored in by system identification. Experimental results were compared with the predicted results calculated from mathematical software. Such a comparison between actual measurements and analysis by modeling of ankle joints for stroke subjects may make it possible to quantitatively evaluate functional recovery from spasticity.
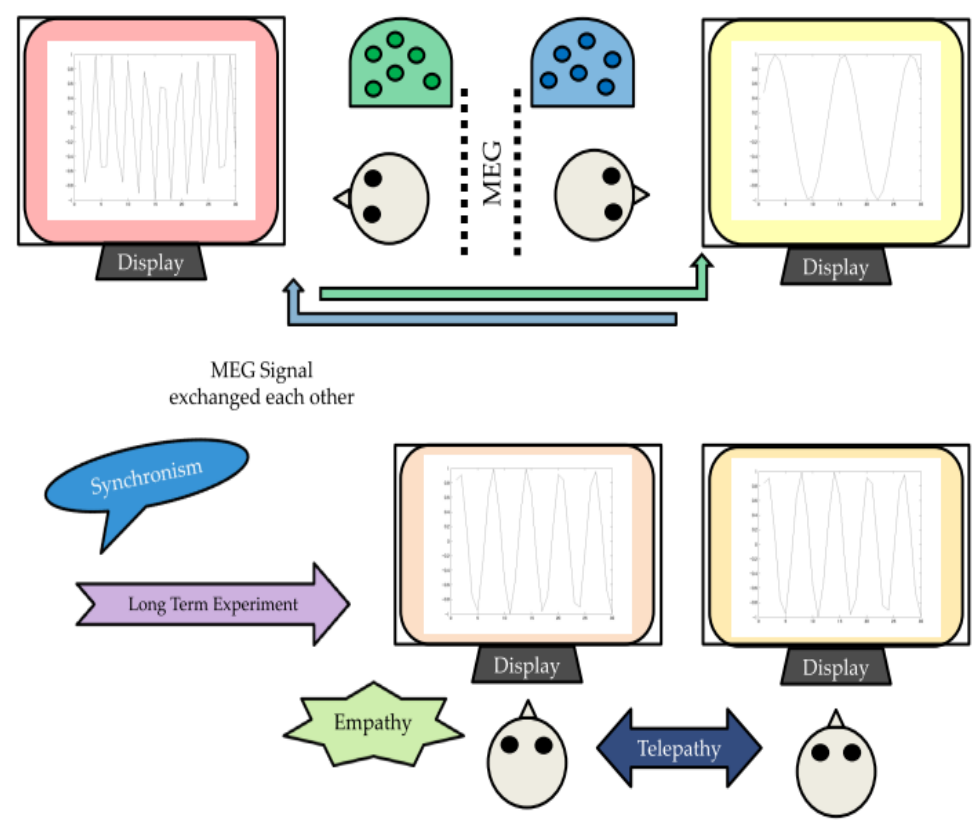

Fig. 2 Neural correlation about inclusive education with communication skill. 


\section{Results and Discussion}

\subsection{Device Development}

Treatment of the nervous system is a common research topic in stroke rehabilitation. It is necessary to approach the nervous system with ideas from neural science when considering the conditions of patients with stroke. However, the nervous system is not visible to the naked eye. Methods to estimate changes in the nervous system that result from functional recovery include resistive torque and electromyographic activities. Another method is modelling the nervous system function, which can be easily acquired with feedback from subjects. Examples of such biomechanical models include Maxwell, Voigt and Kelvin models. Biomechanical models that represent elastic and viscous components are often used, except in biomedical engineering. However, these models should function as multipurpose tools. Biomechanical models that include an inertial component are suggested for modelling muscle activity.

The relationship between the muscle force and length is represented by elasticity, whereas the relationship between the muscle force and rate of shortening is represented by viscosity. The neural reflex is represented by inertia. Biomechanical models represented by viscoelasticity and inertia are used for understanding the ankle joint in healthy subjects. In patients with spinal cord injuries or stroke, the ankle joint is modelled from the point of view of rehabilitation, and it is represented by a state equation. In addition to viscoelasticity and inertia, other components should be included in these models to improve an understanding of abnormal conditions of the central and peripheral nervous systems. These models are based on the hill model, which is based on the spring and damper system and are represented by a transfer function that considers the relationship between input and output signals.

Spasticity is defined as increased resistance to passive motion. Therefore, viscoelasticity in the ankle joint and nervous system are related. Biomechanical evaluation of spasticity is the measurement of viscoelasticity at the ankle joint. Many studies have measured both electromyographic activity (device shown in Fig. 3) and viscoelasticity (device shown in Fig. 4). Modelling of different muscle characteristics should quantify the changes that occur because of rehabilitation. Muscle characteristics can be modelled using different methods. Modelling, which is consistent with the measurement methods, and results are necessary. It is possible to estimate the functional recovery of grasp by modelling the conditions for patients with stroke undergoing rehabilitation. A model should indicate a relationship between the transfer function and differential equation.

Active movement using the brain is called neurorehabilitation. During rehabilitation, a resistive torque and an electromyogram of patients gradually decrease or increase (change), indicated in Fig. 5. These quantitative evaluations of the human body will improve, if numerical modeling based on time series data is developed.

Have you become older, it is a stroke by a factor of lifestyle-related diseases. A factor of lifestyle-related diseases is, for example, insufficient intake of green vegetables and fish. Further, it is evident also onset of stroke due to environmental destruction. Therefore, some sort of treatment is considered as needed. It has been done to represent the recovery by exercise training in the internal model of the cerebellum.

Stroke, or clogged blood vessels in the brain, and torn, it reached its destination cell nutrition, sick cells to die. What happens to temporarily clogged vessels in the cerebral cortex can be treated before a major attack by early detection. Control was filled portion of the brain that the body part to work. Start rehabilitation as soon as possible, to prevent muscle stiffness out complications. By the early start of treatment and rehabilitation, the effect is definitely up. 


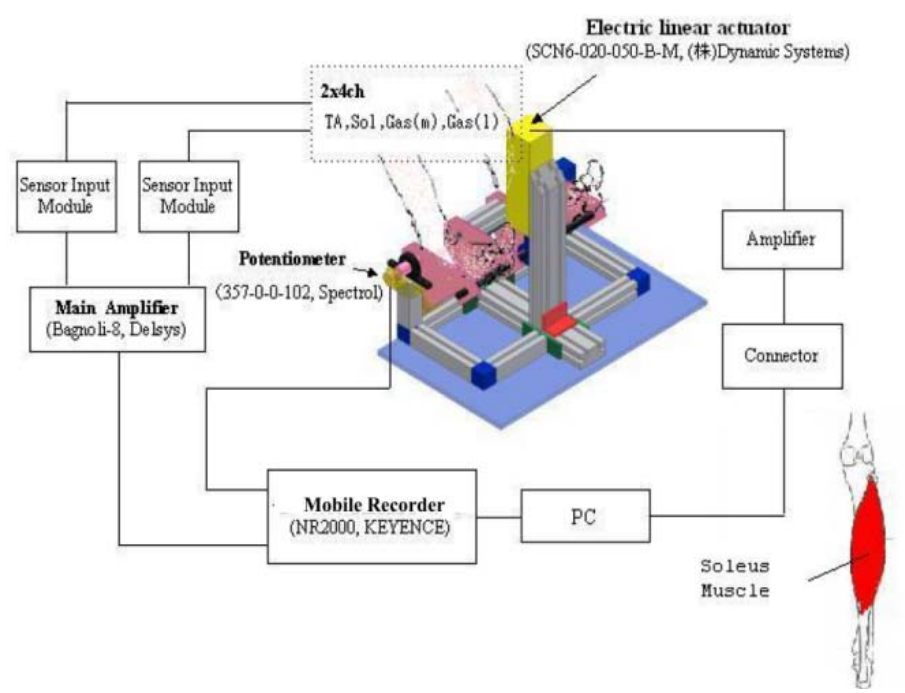

Fig. 3 Neurorehabilitation device with passive movement to Ankle Joint.

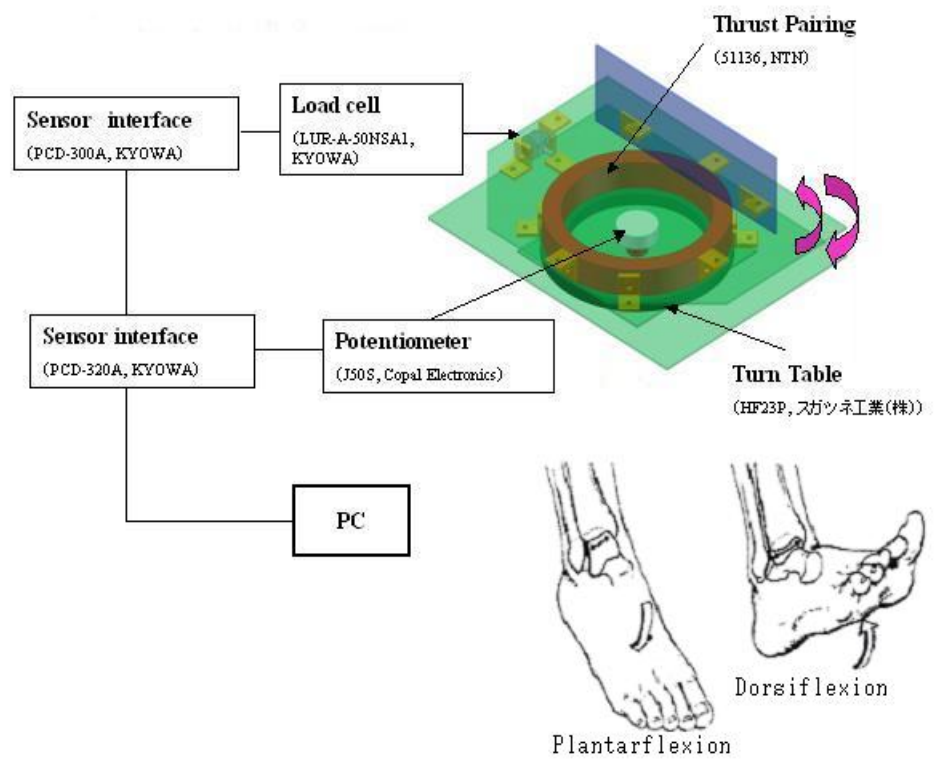

Fig. 4 Torque measurement for effect evaluation of passive movement.

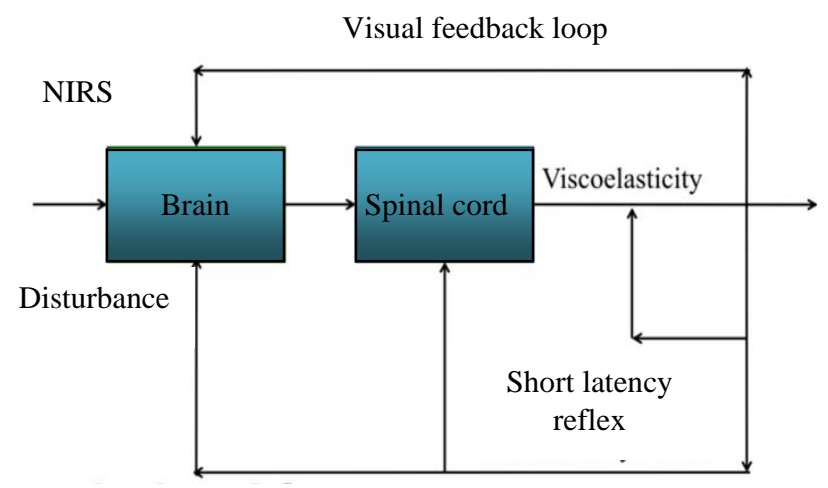

Long latency reflex

Fig. 5 Risk assessment against environmental problems.
Neurorehabilitation was developed by physiotherapists as Bo-bath approach. Take advantage of the central nervous system plasticity of the brain or spinal cord, which aims to improve the treatment capability of people with neurological disease.

From the effects of biological tasks, impaired self-support, and aims to achieve the handicapped individualized. By this, telemedicine research gets help. In home economics education, family care for the aged on smart community was practiced in the class. Longitudinal home care based on subliminal 
effect or hidden curriculum should be evaluated by numerical modeling of stretch reflex. Motor function is connected with sensory function, and so it was aimed to genetic training for brain injuries.

\subsection{Modeling}

Galiana et al. suggested using third-order differential equations for the ankle joint of spinal cord-injured subjects and stroke subjects from a pseudo-random binary sequence [8]. Time delay acts as an exponential function in third-order model by Galiana et al. and is characterized by physiologically short-latency reflex and long-latency reflex, shown in Fig. 6.

Short and long-latency reflexes are indicated by the analysis of Average Rectified Value (ARV). The average rectified measuring data was calculated. The silent period from the onset of passive motion to the onset of the short-and long-latency reflexes varies between muscles and velocity of passive motion [9].

By using appropriate models, numerical analysis, system design simulation technique and the control design, a biological system was developed. If the system structure is particularly evident, the model is described by a formula based on the physical laws governing the subject. This is a physical modeling (modeling white) is called.

In contrast, the entire system or part time is unknown; there is a method of estimating the model from measured input-output data. This is called system identification.

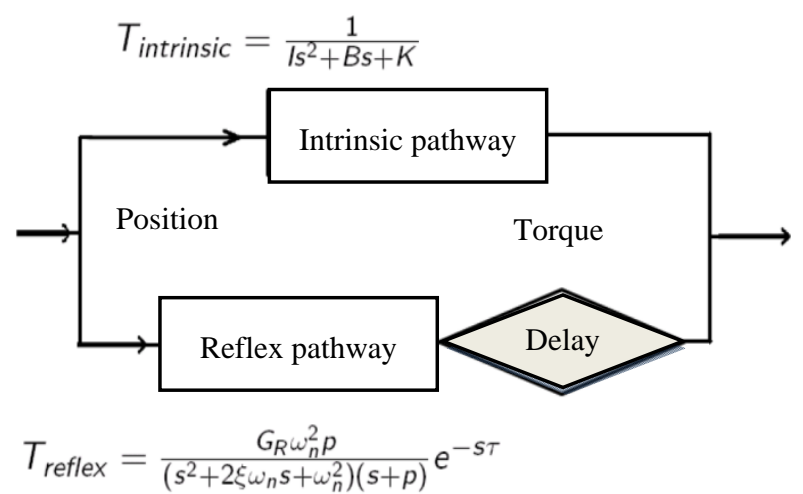

Fig. 6 System diagram with stretch reflex for plasticity of brain function.

\subsection{Analysis Method}

Using software, the Maxima is an open source, "Lisp" was developed. The least square method was used for calculation. The differential equation representing the lower limbs system rewritten in Laplace variable and was determined by inverse Laplace transform. Repeat this flow chart, it is encouraged to be as small as possible a minimum mean square error.

The system structure and, if you have more input and output data is obtained, using a model that reflects the system structure identification, the model estimated by curve fitting for Figs. 7 and 8 .

MATLAB, the Math Works Inc., and the compilation of the correlation function. System structure is unknown, is obtained only if the input and output data, the ARMAX and ARX identification model and assuming a polynomial model, is estimated by the optimization model.

\subsection{Training Menu}

And healthy for one person, three planned training. First one is walking. Second one is jogging. And third one, the stepping (jumping) is each task, so that the treatment of sequelae of hemiplegia or diplegia [10].

Walking and jogging speed ratio is about one to five respectively. The jogging speed is even given the task of stepping. When stressed by environmental, exercise training is required. Effect of exercise training is represented by the control system. For nutrition therapy and exercise training in food education, all training should be picked up.

\subsection{Study Analysis Software}

From data by two-wavelength of brain activation, 2D (two-dimensional) and 3D (three-dimensional) mapping (see in visible light) is possible. When imaging, the maximum measured data, relative to the minimum, the reaction is also expected to be slight catch. 


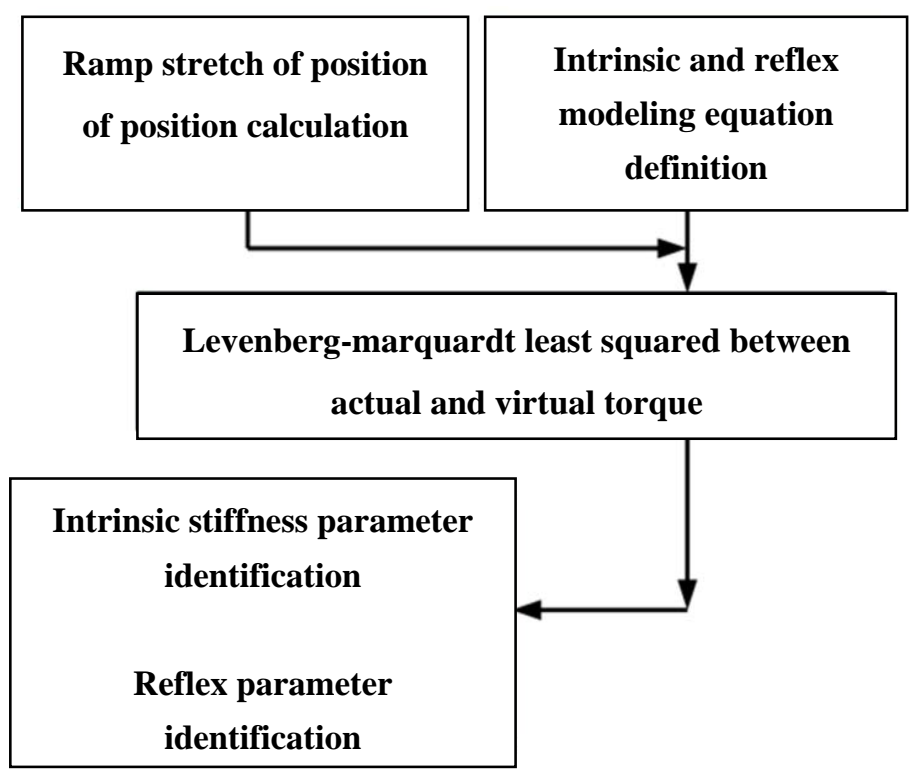

Fig. 7 Flowchart of system identification.

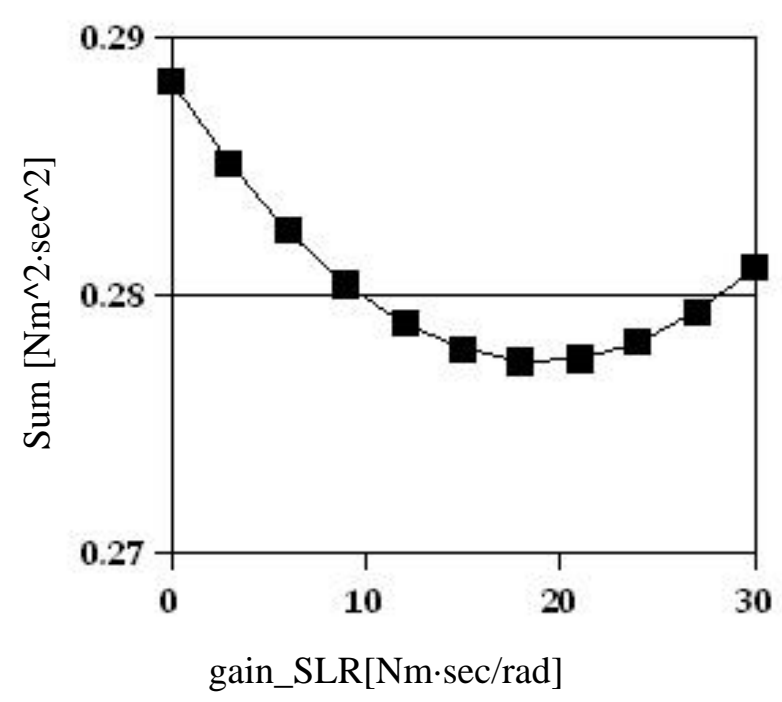

Fig. 8 Short latency reflex gain identification.

For the acquisition of skills, a study was performed on the construction of the learning model [11]. Upon receiving the results suggest improvement of physical function by rehabilitation training model was developed based on a change due to movement of the stretch reflex. The training model is that shown by the adaptive control theory consisting feedback from the feed-forward. Error function is measured as the hysteresis shows the case of stroke in this learning model [12]. By going to reduce the error that is exposed to the hysteresis, it is to be closer to the state of normal subjects.

The appropriate training makes beautiful performance by plasticity and this biological system, Brain model [13] in Figs. 9 and 10, is called as Neurorehabilitation. Curriculum development using subliminal effect is intended to improve the performance of the students. Improving the performance of students has the potential to be quantified.

\section{Conclusions}

\subsection{Behavior Improvement}

An indicator of functional recovery from spasticity is necessary for neurorehabilitation [14], for example modelling the ankle joint. Measurement of electromyogram and resistive torque is important for gender education of home economics.

Elasticity, viscosity, inertia and reflex gain are all decreased by passive motion for acute stroke subject using internal modelling of cerebellum. Specially, reflex gain parameter was highly decreased, and this reason is possibly the change of central nervous system. 


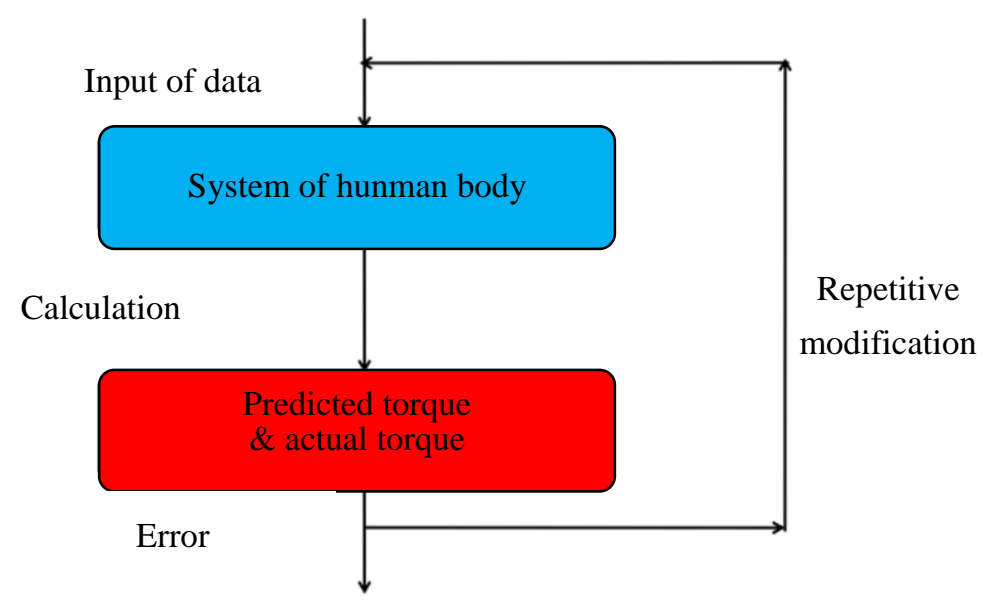

Close to ZERO

Fig. 9 Least squared method between predicted torque and actual torque.

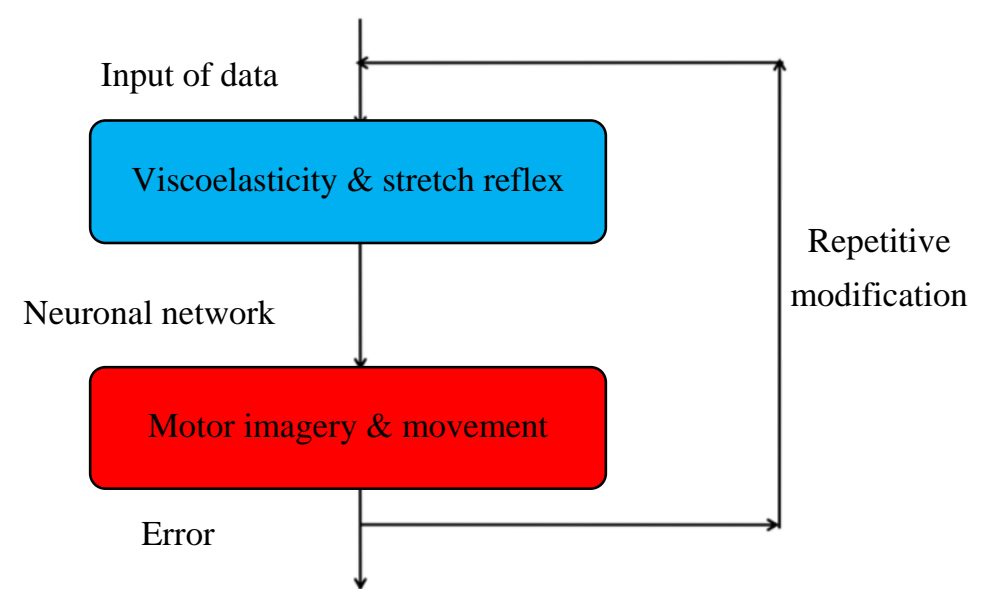

Close to ZERO

Fig. 10 Least squared method between motor imagery and movement.

\subsection{Applications}

Biomedical engineering should be applied to develop human's life in home economics education, which aims children's development by neuroplasticity. This biomedical engineering suggests some technical methods to clinical medicine, by physiology, biochemistry, structural mechanics, architectural design, polymer science and colloid chemistry. The example of such biomedical engineering is neurological rehabilitation, which aims neural recovery of neurological diseases. Home economics makes the children to think about their life from the viewpoint of engineering nowadays. Through research and development of biomedical engineering, education about neurological rehabilitation in home economics would be suggested. Measurement and analysis of neurological rehabilitation, which aims functional recovery by neuroplasticity [15], are like follows. Passive movement is useful for the quantitative diagnosis of people, who have spasticity. Electromyogram, the quantitative diagnosis method, shows two different silent periods in stretch reflex. Those are short latency stretch reflex thorough spinal cord, and long latency stretch reflex through cortex. Also, it revealed from internal model of cerebellum [16], that the function of viscoelastic lower limb, which have spasticity, improves by long latency reflex. And active movement is useful for treatment of 
spastic limb. Biomedical engineering, such as neurological rehabilitation, is science of human's life in home economics. Biomedical engineering needs such educational applications.

\section{Acknowledgments}

The author would like to thank Enago (www.enago.jp) for the English language review. This is collaboration with the University of Tokyo.

\section{References}

[1] A. Gal, G. Szilagyi, E. Wappler, G. Safrany, Z. Nagy, $\mathrm{Bcl}-2$ or Bcl-XL gene therapy reduces apoptosis and increases plasticity protein GAP-43 in PC12 cells, Brain Research Bulletin 76 (4) (2007) 349-353.

[2] S. Heuninckx, N. Wenderoth, S.P. Swinnen, Systems neuroplasticity in the aging brain: recruiting additional neural resources for successful motor performance in elderly persons, Journal of Neuroscience 28 (1) (2008) 91-99.

[3] A.V. Kalue, Neurobiology of memory and anxiety: from genes to behavior, Neural Plasticity. [Online early access]. DOI: 10.1155/2007/78171, Published Online: Jan. 10, 2007, http://dx.doi.org/10.1155/2007/78171 (accessed Jan. 10, 2007).

[4] F. Meintzschel, U. Ziemann, Modification of practice-dependent plasticity in human motor cortex by neuromodulators, Cerebral Cortex 16 (8) (2006) 1106-1115.

[5] U.V. Nagerl, G. Kostinger, J.C. Anderson, K.A.C. Martin, T. Bonhoeffer, Protracted synapto-genesis after activity-dependent spinogenesis in hippocampal neurons, Journal of Neuroscience 27 (30) (2007) 8149-8156.

[6] J.J. Ross, C.M. Verfaillie, Evaluation of neural plasticity in adult stem cells, Philosophical Transactions of the
Royal Society B: Biological Sciences 363 (1489) (2008) 99-205.

[7] F.A.J.L. Scheer, Jr.K.P. Wright, R.E. Kronauer, C.A. Czeisler, Plasticity of the intrinsic period of the human circadian timing system, PLoS ONE. [Online early access]. DOI: 10.1371/ journal.pone.0000721, Published Online: August 08, 2007, http://www.plosone.org/article/ info\%3Adoi\%2F10.1371\%2Fjournal.pone.0000721 (accessed Dec. 8, 2004).

[8] L. Galiana, J. Fung, R. Kearney, Identification of intrinsic and reflex ankle stiffness components in stroke patients, Experimental Brain Research 165 (2005) 422-434.

[9] S. Michael, C. Stephanie, C. Leonardo, D. Pamela, G. Fred, Textbook of Neural Repair and Rehabilitation, Cambridge University Press, London, 2006.

[10] J. Bovend'Eerdt, M. Newman, K. Barker, H. Dawes, C. Minelli, D.T. Wade, The effects of stretching in spasticity: a systematic review, Archives of Physical Medicine and Rehabilitation 89 (7) (2008) 1395-1406.

[11] S. Dong, K.Q. Lu, J.Q. Suna, K. Rudolph, Rehabilitation device with variable resistance and intelligent control, Medical Engineering and Physics 27 (3) (2005) 249-255.

[12] P.Y. Lin, Y.R. Yang, S.J. Cheng, R.Y. Wang, The relation between ankle impairments and gait velocity and symmetry in people with stroke, Archives of physical medicine and rehabilitation 87 (4) (2006) 562-568.

[13] D.G. Kamper, B.D. Schmit, W.Z. Rymer, Effect of muscle biomechanics on the quantification of spasticity, Annals of Biomedical Engineering 29 (12) (2001) 1122-1134.

[14] F.M. Vaccarino, Y. Ganat, Y. Zhang, W. Zheng, Stem cells in neurodevelopment and plasticity, Neuropsychopharmacology: Official Publication of the American College of Neuropsychopharmacology 25 (6) (2001) 805-815.

[15] C. Vaillend, R. Poirier, S. Laroche, Genes, plasticity and mental retardation, Behavioural Brain Research 192 (1) (2008) 88-105. 